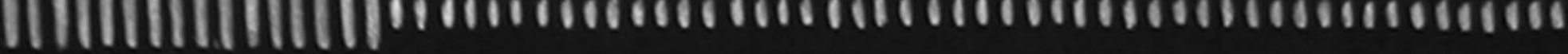

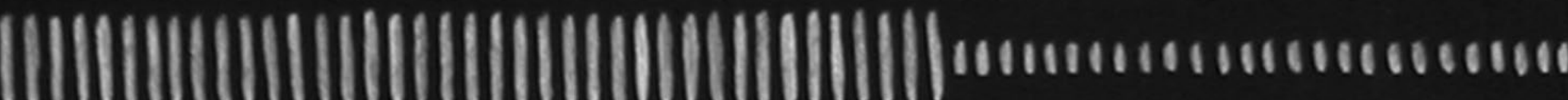

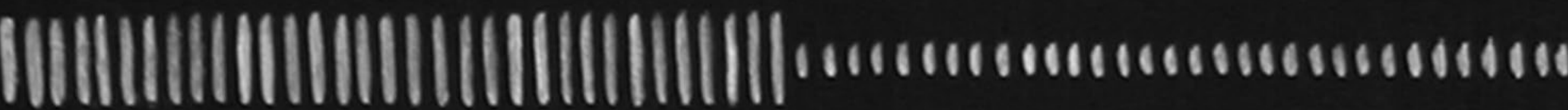

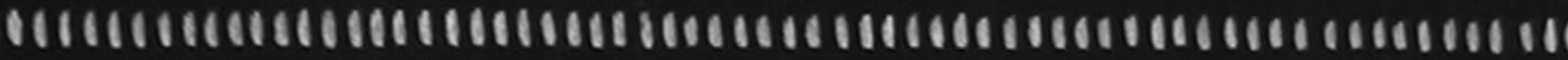

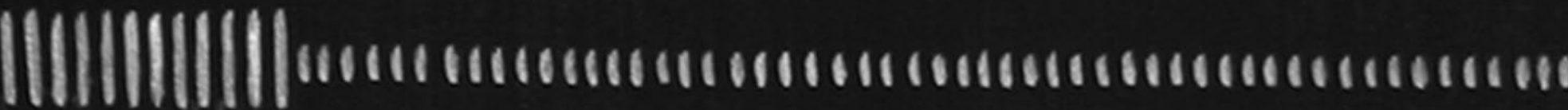
|||||||||||||||||||||||||||||||||||||||||||||||||||||||||||||||||

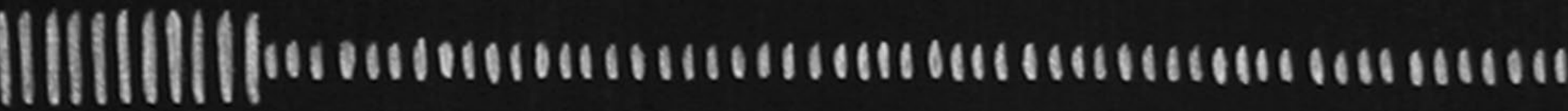

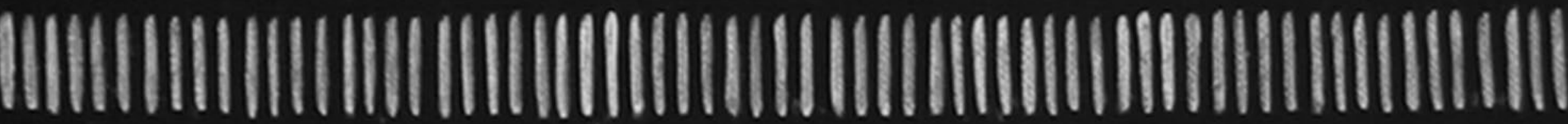
|||||||||||||||||||||||||||||||||||||||||||||||||||||||||||||||||||

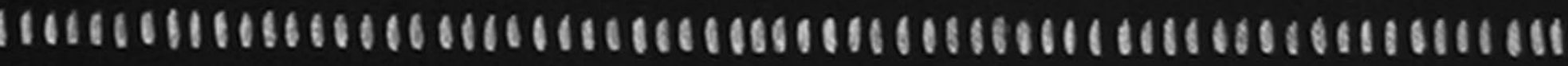

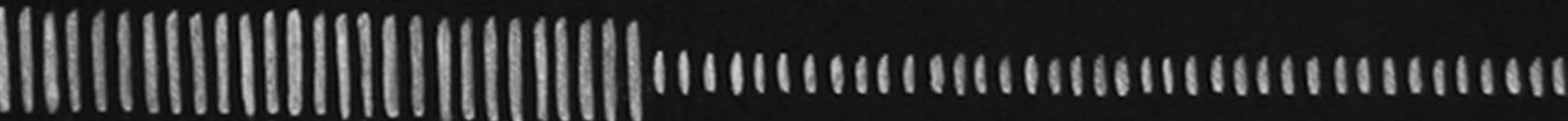




\title{
JUSTICIA INDÍGENA EN TIEMPOS MULTICULTURALES: EL CASO DEL JUZGADO INDÍGENA DE CUETZALAN, PUEBLA EN MÉXICO
}

\author{
Indigenous justice in multicultural times: \\ The case of the Indigenous Court of Cuetzalan, Puebla in Mexico
} ADRIANA TERVEN SALINAS*

Fecha de recepción: 22 de julio de 2014 - Fecha de aprobación: 5 de septiembre de 2014

\section{Resumen}

A partir del reconocimiento constitucional de la diversidad cultural en México, las relaciones entre el Estado y los pueblos indígenas parecen tomar un cauce distinto a como habían venido configurándose, basadas en relaciones de poder y discriminación. No obstante, detrás del discurso multicultural se siguen reproduciendo representaciones sociales que otrora fueron legitimadas por las políticas indigenistas planteando retos para el cabal reconocimiento de derechos culturales. El presente artículo pretende evidenciar desde la experiencia del juzgado indígena de Cuetzalan, Puebla, cómo el proyecto estatal mantiene una misma intención: asegurar la permanencia del modelo monocultural de Estado, así como atender los nuevos imperativos internacionales de reforma estructural.

Palabras Clave: indigenismo, justicia y derechos culturales

\section{Abstract}

From the constitutional recognition of cultural diversity in Mexico, the relations between the state and indigenous peoples, seem to take a different path as they had been taking shape, based on power relations and discrimination. But behind the multicultural discourse there are social representations, that once were legitimized by indigenous policies, which pose challenges for the proper recognition of cultural rights. This article aims to show the experience from the Indian court in Cuetzalan, Puebla, how the state project maintains the same intent: to ensure the permanence of the state monocultural model, as well as meet new international imperatives of structural reform.

Key words: Indigenous policy, justice, cultural rights

* Doctora en Antropología. Universidad Autónoma de Querétaro (México). Correo electrónico: adrianaterven@yahoo.com.mx 


\section{Introducción}

"Es la primera vez que veo tanto apoyo para los indígenas" decía un nahua de la Sierra Norte de Puebla mientras contemplaba el nuevo edificio del juzgado indígena en la cabecera municipal de Cuetzalan; "ahora si nos están tomando en cuenta", concluyó su frase. Expresiones como estas se han escuchado para hacer referencia a las políticas de estado relacionadas con los pueblos indígenas en el actual contexto del Estado multicultural, pareciendo marcar una importante distinción con la manera en cómo el Estado había venido representando e interviniendo a este sector de la población, caracterizadas por la discriminación de sus culturas y la marginación. Efectivamente el reconocimiento de la diversidad cultural ha planteado la posibilidad de generar relaciones sociales que descansen en el respeto por la diferencia y su inclusión bajo este mismo principio, no obstante los imaginarios sociales que otrora modelaron las relaciones entre indígenas, Estado y sociedad parecen prolongarse generando contradicciones entre los discursos a favor de los derechos culturales y las acciones que de ellos se desprenden.

Como veremos en este texto, en México el desprecio por las culturas indígenas ha pasado de su expresión e intervención explícitas por erradicarlas, a un discurso que promueve su respeto y conservación. Tal y como se deja ver en el testimonio inicial, el juzgado indígena ha representado para la población local el reconocimiento y apoyo material a sus instituciones. Hasta antes de este juzgado, los jueces tradicionales habían venido ejerciendo sus labores bajo condiciones precarias y el desdén de las autoridades estatales; el juzgado indígena, construido y equipado a imagen de las oficinas de la ciudad capital, "parecía otorgar de manera mágica", un nuevo lugar a la población indígena dentro de las relaciones locales, históricamente marcadas por la discriminación, el despojo y la violencia.

Es la ilusión del nuevo pacto social lo que pretendo mostrar a continuación, evidenciando la manera en cómo los discursos multiculturales se traducen en acciones, muchas de las veces, contradictorias. En particular analizó la implementación de la reforma judicial en materia indígena realizada por el Estado de Puebla, mostrando como ciertas representaciones sociales legitimadas por las políticas indigenistas continúan permeando, y cuya intención es la misma: asegurar la permanencia del modelo de estado monocultural, así como atender los nuevos imperativos internacionales de reforma estructural.

Presento cuatro apartados, en el primero refiero las políticas dirigidas a los pueblos indígenas, cuyos objetivos buscaban acabar con la diversidad étnica y cultural, siendo así la plataforma donde se construyeron ciertas representaciones sociales respecto de este grupo poblacional; posteriormente abordo las nuevas políticas indigenistas, en las que el reconocimiento multicultural de la nación, plantea nuevos retos para las relaciones entre el Estado y la población indígena bajo el velo del respeto por la diversidad cultural. Este contexto político y su problematización son retomados en el apartado tres, expresado en el caso de estudio de la reforma judicial poblana y la instalación del juzgado indígena en Cuetzalan. 
El análisis presentado es parte de los resultados de mi investigación de doctorado (Terven, 2009), en la cual trabajé durante más de cuatro años los procesos organizativos locales relacionados con el acceso a la justicia y las prácticas de justicia indígena, temas que componen el contenido del cuarto apartado, el cual aborda lo que han sido los movimientos sociales de Cuetzalan, en específico su participación en el juzgado indígena.

\section{Indigenismos}

Históricamente, el Estado mexicano y los pueblos indígenas, han mantenido relaciones políticas organizadas bajo distintas lógicas llamadas genéricamente como indigenismos, cuyos principales propósitos han tenido que ver con incorporar al indígena a la sociedad nacional, buscando con ello acabar con la heterogeneidad étnica a favor de una paulatina homogenización de la sociedad. En este apartado se presentan los principales argumentos e intenciones que dieron forma a las políticas indigenistas en México, evidenciando los imaginarios sociales que han modelado las relaciones entre los pueblos indígenas con el Estado y con la sociedad.

De acuerdo con Díaz-Polanco (2004), se reconocen tres políticas indigenistas importantes en México: la incorporativista, la integracionista y la etnopopulista. Durante el periodo colonial igualmente se implementaron políticas dirigidas a la población nativa denominadas como segregacionistas. Respecto de este momento quizás valga la pena mencionar su objetivo, el cual buscaba separar a los indígenas de la sociedad europea por medio de mecanismos basados en la discriminación racial, la dependencia económica, el control político, la distancia social, entre otros, lo que creó una barrera étnica que dividió a la sociedad en castas. Si bien con el México independiente, las políticas relacionadas con los pueblos indígenas parecían dejar atrás estos sesgos racistas, a continuación mostramos lo que ha sido un continuo de relaciones de desigualdad oficializadas por el Estado.

La política indigenista incorporativista a diferencia de la segregacionista, se caracterizó por la incorporación de los grupos indígenas a la nación. Fue impulsada a mediados del siglo XIX, junto con la política de Reforma dirigida por el presidente Benito Juárez, quien se basó en las ideas liberales que privilegiaban los derechos individuales promoviendo la libre competencia, la ganancia y la propiedad privada, beneficiando a grandes hacendados en detrimento de las bases materiales tanto de los grupos étnicos como de la iglesia. Fue así que para conseguir la incorporación del indio a la nación y conservar los ideales occidentales, se prohibieron las costumbres, creencias y formas de vida indígena, en otras palabras, no admitía particularidades (Díaz-Polanco 2004; Villoro, 1996).

A principios del siglo $X X$, se ubica una tercera política indigenista llamada integracionista, la cual forma parte de las ideas de Manuel Gamio, quien señalaba que en México no existía un auténtico patriotismo nacional debido a la diversidad regional, étnica y local, proclamando que era necesario que los indígenas se integraran a la nación. En sus postulados se observa la influencia del pensamiento evolucionista y ciertos matices del relativismo cultural, dado que organiza a la sociedad en etapas civilizatorias y ubica a los 
grupos étnicos en las inferiores, no obstante planteó la integración de tal modo que se respetaran e incluyeran, hasta cierto punto, valores positivos del sistema sociocultural indígena (Oehmichen, 1999; Villoro, 1996).

Un segundo momento de esta política estuvo a cargo de Gonzalo Aguirre Beltrán, quien introdujo la noción de regiones de refugio. Sostuvo que México no surgió como nación homogénea pues estaba dividido en dos grupos socioculturalmente opuestos, los indígenas y los ladinos, fue así que los procesos de forja de la nación caracterizados por el despojo y la violencia hacía los primeros, terminó por desplazarlos a ciertas áreas, las cuales denominó como regiones de refugio (Díaz-Polanco, 2004). El acontecimiento que selló esta tendencia fue el Congreso Interamericano de Pátzcuaro en 1940:

\footnotetext{
"El contenido de propósito de la política indigenista formulada en Pátzcuaro era la integración del indio a la sociedad nacional, con todo y su bagaje cultural, proporcionándole los instrumentos de la civilización necesarios para su articulación dentro de una sociedad moderna" (Aguirre, 1984:22).
}

La propuesta radicaba en que los grupos indígenas mantuvieran sus particularidades y al mismo tiempo aceptaran los sistemas e instituciones nacionales que definen las formas de vida nacional. Lo que interesa rescatar de estas políticas indigenistas, en especial las dos últimas correspondientes al México independiente, es el tipo de relación social que promovieron entre la población indígena y la sociedad nacional. En este sentido, observamos cómo el Estado ha venido legitimando relaciones de desigualdad, discriminación, despojo y violencia, las cuales como pretendemos mostrar se mantienen a lo largo del tiempo, pasando de un discurso político explícito como ya vimos, a un discurso que bajo el velo del respeto a la diversidad cultural, el llamado neo-indigenismo parece incluso volverse más abrasivo.

Con base en lo anterior, podemos decir que la representación social que se construyó de este sector como sociedades inferiores, lo exhibe como incapaz de manejar sus asuntos, de ahí que a la fecha el tipo de relación que guía el imaginario social ubique a la población indígena en calidad de receptora de programas y apoyos de gobierno, y de beneficencia pública, e incompetente para generar y llevar a cabo alternativas de desarrollo, educación, salud, justicia, gobierno, etc. Tal y como argumentó Aguirre Beltrán:

\section{"(...) la política indigenista no es la que el indio formula en lo que concierne a su propia comunidad, sino la manera como el grupo nacional contempla el tratamiento que debe dar a los grupos llamados indígenas de acuerdo con los valores y los intereses nacionales. (...) El indio, como tal, no puede postular una política indigenista porque el ámbito de su mundo está reducido a una comunidad parroquial, homogénea y preclasista que no tiene sino un sentido y una noción vagos de la nacionalidad" (Aguirre, 1984: 24).}

Otro aspecto que interesa resaltar es el concepto de regiones de refugio, pese a que hoy en día se considera como una unidad de análisis insuficiente ya que: "la estructura que se observa a escala regional, no puede ser comprendida a fondo si no se tiene en cuenta que ella forma parte de una unidad mayor: la formación socioeconómica y política mexicana." (Díaz-Polanco, 2004: 141). No obstante, Ilama la atención cómo actualmente predominan ideas en las que los pueblos indígenas parecieran estar aislados del resto de la sociedad, 
lo cual se refleja, por ejemplo, en las políticas de reconocimiento de la diversidad cultural iniciadas en 2001, en las que el ejercicio de derechos se ha planteado en niveles internos de organización social, "lejos" del resto de la población, situando nuevamente a estos lugares como obstáculos para el funcionamiento y expansión del capitalismo.

Otra corriente indigenista impulsada a medidos de 1970, fue la conocida como etnicista o etnopopulista, que si bien no tuvo la relevancia política que las anteriores, vale la pena mencionarla como parte del análisis de las ideas y representaciones que han mediado las relaciones entre la población indígena, el Estado y la sociedad. Uno de sus principales exponentes fue Guillermo Bonfil, cuya propuesta tendió a ver a las comunidades indígenas como armónicas y no alienadas, a diferencia de la atroz sociedad capitalista. Bajo esta idea, la solución a los problemas de la sociedad nacional se ubicaba en el marco de las primeras, exaltándolas e idealizándolas, cuando en la realidad, las comunidades estaban atravesadas por profundas relaciones inter e intraétnicas de poder y de desigualdad, expresadas en escenas de alta marginación y violencia (Díaz-Polanco, 1994; Oehmichen 1999). No obstante, dicha visión también ha formado parte del imaginario social respecto de los pueblos indígenas, quienes son representados como sociedades de gran nobleza y festividad.

Hasta aquí tenemos un primer esbozo de la manera en cómo el Estado ha construido a este sector de la población, justificando sus intervenciones a partir de una gama de nociones que ubican a los indígenas como sociedades atrasadas, incapaces de resolver sus propios problemas e incompatibles con el proyecto nacional; hasta aquellas que ubican en sus rasgos culturales principios positivos e incluso necesarios para resolver los dilemas nacionales (Vázquez, 2011). Se trata de una construcción compleja, y como tal, se expresa en las actuales políticas multiculturales, presentando diversos retos para los movimientos indígenas contemporáneos, así como para su estudio y comprensión.

\section{Neoindigenismos}

Si bien la política indigenista ha respondido a distintas necesidades e intereses propios de su momento, fue en la década de los noventa donde podemos ubicar un cambio tajante en sus paradigmas, a partir del reconocimiento políticolegal de la diversidad cultural. Este contexto planteaba una transformación radical en las relaciones entre el Estado y los pueblos indígenas, bajo la promesa de un nuevo pacto social (Sieder, 2002).

Es así que en México se realizaron distintas acciones en la materia, como fue la ratificación del Convenio 169 de la Organización Internacional del Trabajo (OIT) en 1990, y la reforma al artículo cuarto constitucional, en 1992, que reconocía el carácter multicultural de la nación promoviendo la protección y el desarrollo de las culturas, lenguas, usos, costumbres y formas específicas de organización social de la población indígena. Como parte de este clima cultural, en 1994 el levantamiento zapatista de Chiapas dio mayor fuerza a los debates sobre la conformación de naciones plurales, concretándose en la reforma al artículo segundo de la Constitución, en 2001. 
Si bien es cierto que por primera vez se reconoció de manera más específica derechos indígenas, estas reformas resultaron ser bastante restringidas, ya que a pesar de que dicen reconocer derechos, traen consigo candados que limitan e impiden llevar a cabo el real ejercicio de las disposiciones.

Tales limitaciones en gran parte coinciden con los ámbitos de acción económica y política de las comunidades indígenas, lo cual revela la posición del Estado en cuanto a restringir las capacidades de negociación de estos pueblos para la implementación y gestión de proyectos en conjunto o con sectores como el privado. No obstante lo limitado de este marco legal nacional, y bajo lo que se empezó a considerar como lo políticamente correcto, se han impulsado reformas legislativas en materia indígena en diversas entidades federativas del país, como sucedió en el Estado de Puebla1. A continuación presentamos el caso poblano desde la experiencia del municipio de Cuetzalan, ya que consideramos que muestra en gran medida la manera en cómo las actuales políticas indigenistas configuran las relaciones entre el Estado y la población indígena, en el marco del reconocimiento de la diversidad cultural. En este sentido, me interesa explorar qué tanto se siguen reproduciendo aquellas representaciones sociales construidas en los indigenismos anteriores a las políticas multiculturales, o en todo caso, advertir en los nuevos mecanismos que perpetúan las relaciones de poder y discriminación.

En especial, no enfocaremos en las reformas realizadas en el campo de la justicia, como fue la creación de los juzgados indígenas, en 2002, por el Tribunal Superior de Justicia del Estado, siendo en el municipio de Cuetzalan donde se abre el primero de ellos.

Estos juzgados fueron incluidos como parte de las instancias en las que deposita el ejercicio del Poder Judicial, como lo indica su Ley Orgánica, mediante una reforma en este mismo año. En 2005, la reforma al Código de Procedimientos Civiles reconoció a las prácticas, usos, costumbres, tradiciones y valores culturales de los pueblos y las comunidades indígenas como medios alternativos de resolución de conflictos. Todas estas reformas, plantean distintas implicaciones en la organización del campo jurídico regional, de aquí la importancia por ver la manera en cómo se articulan con las prácticas de justicia locales.

Ahora bien, para abordar el tipo de representaciones sociales presentes en la formulación e implementación de dichas disposiciones legales, nos concentraremos en indagar: ¿En qué medida podemos hablar de un reconocimiento de la diversidad cultural por parte del Poder Judicial?, es decir, ¿qué tanto se reproduce la lógica y organización hegemónica del Estado?; o por el contrario, ¿qué capacidad tendría como un recurso para alcanzar la autodeterminación?

\section{Entre el reconocimiento y la oficialización. La política poblana en materia de justicia indígena desde Cuetzalan, Puebla.}

El municipio de Cuetzalan se ubica en lo que se conoce como la Sierra Norte, que forma parte de la cadena montañosa de la Sierra Madre Oriental. Es un municipio compuesto por un $80 \%$ de población nahua, de un total de 45,000 habitantes aproximadamente. 
Junto con una gran riqueza natural que caracteriza la región, Cuetzalan se sitúa dentro de los niveles socioeconómicos más bajos del país. La actividad principal de la mayoría de las familias es la agricultura de subsistencia, siendo el maíz, el fríjol, el jitomate, chile verde y chile seco los principales cultivos ${ }^{2}$.

La cabecera municipal de Cuetzalan concentra el control político y judicial de la región, constituyendo el engranaje principal entre el Estado de Puebla con las comunidades que integran el municipio, habitadas en su mayoría por población nahua. Cuetzalan representa un centro de poder rural, caciquil mestizo arraigado en la zona desde el siglo XIX, pero también es un espacio marcado por las prácticas y costumbres indígenas. Un aspecto que es relevante destacar, es que Cuetzalan también se ha caracterizado por tener un fuerte proceso organizativo indígena desde la década de 1980.

De entre las organizaciones locales, hay tres que me interesa destacar, la Comisión de Derechos Humanos Takachiualis (respeto mutuo), la Maseualsiuamej Mosenyolchicauanij (Mujeres indígenas que trabajan juntas) y el Centro de Asesoría y Desarrollo entre Mujeres (CADEM), las cuales se han centrado en promover la defensa de los derechos humanos, derechos de las mujeres, y recientemente, derechos culturales. A raíz de la instalación del juzgado indígena en 2002, integrantes de estas organizaciones iniciaron acciones de manera conjunta para fortalecer sus sistemas normativos, ante la necesidad de posicionar sus prácticas propias de justicia frente a la definición que el Poder Judicial de Puebla estaba haciendo de esta. Como veremos a continuación, la reforma en materia indígena más que reconocer las prácticas vigentes de justicia indígena, oficializó una versión de éstas que resultó ser compatible con el modelo judicial del Estado y con las necesidades de reforma estructural que justo se realizaban en ese momento.

Para dar cuenta del proyecto multicultural del Estado, llevé a cabo un análisis de la construcción discursiva del Poder Judicial de Puebla y retomé la propuesta de Gupta (2006) para observar la manera en cómo el Estado poblano se representa así mismo ante la sociedad, en relación con sus procesos de modernización que integran, entre otros, el tema indígena. Realicé una amplia revisión de distintos documentos como fueron la gaceta del Poder Judicial, Acuerdos de Pleno y Nombramientos emitidos por el Tribunal Superior de Justicia, la Ley Orgánica del Poder Judicial y el Código de Procedimientos Civiles $^{3}$; esta producción discursiva, refleja en buena medida el tipo de relación que el Estado poblano ha construido con los pueblos indígenas ubicados en su entidad, la cual nos muestra las representaciones sociales que median sobre este sector de la población.

Un primer documento que me interesa presentar relacionado con la reforma judicial en materia indígena, fue la noticia publicada en la revista del Tribunal Superior de Justicia (TSJ), que llevaba por título: "Banco Mundial apoya al Poder Judicial del Estado de Puebla", en la que se reseña el "Taller Hacía la Modernización Judicial", realizado en abril de 2003 en el TSJ. Según la nota, en este taller participó un representante del Banco Mundial para América Latina. El taller "dio paso a una serie de propuestas para mejorar la calidad del servicio, la atención al público, la reducción de tiempo para los procesos judiciales" 4 . 
Asimismo, se enfatizó la constante revisión de las leyes para actualizarlas y mejorarlas, destacándose la reforma de la Ley Orgánica en 2002, que amplió las competencias -e incluyó a los juzgados indígenas- generando con esto, mayor acceso de los ciudadanos a la justicia.

Sierra (2004), menciona que eventos como éste, revelan la necesidad del Estado de adecuar las instituciones judiciales a las exigencias actuales de impartición de justicia a nivel internacional, así como a los procesos de descentralización federal y estatal. Santos (1999), refiere que la transnacionalización de la regulación jurídica del Estado-nación, responde a

\footnotetext{
“(...) cualquier situación en la que se pueda establecer que los cambios en el derecho estatal de un país dado han sido influidos decisivamente por presiones internacionales, formales o informales, de otros estados, agencias internacionales $u$ otros actores transnacionales. Tales presiones tienden a ser ejercidas (...) en diferentes partes del sistema interestatal" (lbídem: 80).
}

En el caso de Puebla, la modernización judicial, en cierta medida ha sido impulsada por instituciones multilaterales como lo fue el Banco Mundial; llama la atención cómo en este mismo momento, en 2002, el TSJ creó el Centro Estatal de Mediación (CEM), el cual representa un claro ejemplo del proceso de transnacionalización del campo jurídico. Con el CEM el Tribunal instituyó los métodos alternativos de resolución de conflictos (MARC), los cuales han sido promovidos internacionalmente como la vía para el acceso y mejoramiento de la justicia como, garantía de la convivencia pacífica.
Cabe mencionar que el modelo de la mediación surgió en Estados Unidos a mediados de 1960, instituido como un medio alternativo, el cual ha sido ampliamente difundido.

El contexto anterior cobra gran relevancia para el análisis, ya que en el Código de Procedimientos Civiles se reconoció a los usos, costumbres, tradiciones y valores de los pueblos indígenas bajo la denominación de método alternativo. Con dicha designación, el Estado poblano niega la vigencia de los sistemas normativos indígenas, lo cual le permite al CEM ser el encargado de instruir el procedimiento de atención y resolución de conflictos en los juzgados indígenas, situación que dicho sea de paso, termina por volver compatible la práctica de la justicia indígena con el proyecto de reforma judicial (Terven, 2009). Acciones como estas definitivamente están permeadas por representaciones del indígena como inferior e incapaz de llevar a cabo sus propios asuntos de justicia, ubicándolo como antagónico al proyecto del Estado moderno, lo cual nos recuerda en mucho las políticas indigenistas anteriores al contexto político multicultural.

En Cuetzalan, esta situación ha provocado encuentros contradictorios entre la práctica de la justicia indígena local con el proyecto estatal de los MARC. EI TSJ al señalar que los juzgados sean atendidos por pobladores indígenas, bajo sus prácticas, usos, costumbres, tradiciones y valores culturales, resta a la justicia indígena su carácter normativo. En el caso de Cuetzalan, el juez indígena, de origen nahua y ex autoridad en su comunidad, se ha visto en la contradicción de llevar a cabo la dinámica de la mediación o las dinámicas de resolución propias del derecho nahua. 
Respecto a la creación de juzgados indígenas, el Acuerdo de Pleno del TSJ del 14 de marzo de 2002 señala que la instauración de estos juzgados pretende atender lo dispuesto en dos artículos Constitucionales, el art. 17 y el art.25 . El primero dicta que todas las personas tienen derecho a que se les administre justicia de manera pronta, completa e imparcial, y el segundo "establece que las personas que integran los pueblos indígenas, además de gozar de dichas garantías constitucionales (art. 17), en la solución y regulación de sus conflictos se aplicarán sus propios sistemas normativos"6.

Sin embargo, el Acuerdo señala que sus "codificaciones en general no atienden claramente a los indígenas dada su propia forma de organizarse y normarse aplicando sus costumbres", de aquí la necesidad de "crear los mecanismos para que dichos grupos tengan órganos jurisdiccionales de fácil acceso y logren así obtener la justicia a que se refiere el mencionado artículo 17"7. En consideración con esto, el presidente del Tribunal estimó pertinente la creación de Juzgados Menores Mixtos para asuntos indígenas, los cuales "podrán utilizar los mecanismos de mediación que establece el Código Procesal Civil, y conforme a los usos y costumbres que estén acorde con la Constitución Política"8.

Como podemos ver, la ambigüedad del discurso en el Acuerdo de Pleno fluctúa entre el reconocimiento de sistemas normativos, pero entendidos como mecanismos de mediación, tal y como están referidos en el Código. Desde esta revisión, nos encontramos con que el proyecto estatal de justicia no consideró la demanda indígena local, incluso algunas organizaciones indígenas como la
Unidad Indígena Totonaca Nahua (UNITONA), tenía propuestas de legislación desarrolladas en base a consultas hechas al interior de sus comunidades. Tampoco consideró las prácticas vigentes de justicia indígena, mostrando así un proyecto de justicia que conserva en mucho una lógica hacia homogeneizar los procedimientos de atención, en vez de reconocer la diferencia y la diversidad.

No obstante lo anterior, el TSJ se ha construido discursivamente como innovador en materia de justicia indígena, discurso que podría ubicarse en lo que se ha llamado el nuevo marco político neoindigenista. Estas políticas suponen la consolidación de un multiculturalismo oficial en México, "que ha logrado articular políticamente la diferencia como parte del orden social, incorporando los motivos y aspiraciones de los oprimidos para volverlos compatibles con las relaciones de dominación" (Hernández, Paz \& Sierra, 2004: 21).

El análisis de discursos oficiales reveló cómo la reforma en Puebla es parte de un marco más amplio de políticas neoliberales de ajuste estructural, las cuales han promovido al apoyo legal como un derecho fundamental de los ciudadanos (Assies, 1999). Las reformas realizadas por el Poder Judicial reproducen el discurso modernizador de "eficiencia" y de acceso a la justicia del contexto internacional, con el fin de descentralizar y modernizar el aparato de justicia. En este sentido, el Poder Judicial poblano, en vez de hacer cambios de fondo en la organización del poder regional para reconocer la jurisdicción indígena, incluyó a los usos, costumbres, tradiciones y valores de los pueblos indígenas como un método alternativo de resolución de conflictos (MARC). 
Desde esta perspectiva, el paso de la justicia indígena a la legislación se da en concordancia con este marco internacional, de ahí que planteo que se trató más bien de una oficialización y no tanto de un reconocimiento, ya que sólo se institucionalizó como "mecanismos informales a través de los cuales, puede resolverse un conflicto de intereses en forma extraprocesal, coadyuvando así, a la justicia ordinaria"'.

Para poder hablar de un reconocimiento, es necesaria una transformación en el sistema jurídico estatal para que la justicia indígena sea incluida como sistema jurídico. El reconocimiento planteado como demanda de autonomía proclamada por los pueblos indígenas de México, responde a la necesidad de integrarse a la política del Estado nacional bajo formas que descansen en la coordinación (Díaz-Polanco, 1996), no obstante en el caso de Puebla, advertimos una integración de la justicia indígena al marco legal que termina por institucionalizar la relación de subordinación del derecho indígena ante el derecho estatal, tal y como ha venido sucediendo en la larga data de indigenismos ${ }^{10}$.

\section{El Juzgado Indígena de Cuetzalan}

El juzgado indígena de Cuetzalan se inauguró en marzo de 2005, tres años después de su apertura, el evento contó con la presencia del gobernador del estado de Puebla y del presidente del TSJ, quienes en sus discursos hablaron de la deuda pendiente con los pueblos indígenas: la justicia, que ahora se subsana con la apertura de este espacio. También estuvieron diversos funcionarios del Poder Judicial, así como el director del CEM, todos ellos rodeados por un nutrido grupo de reporteros.
De acuerdo con Sharman y Gupta (2006), la inauguración representó un momento clave para los funcionarios del TSJ, quienes restituían su poder desde este evento al presentar su versión de la justicia indígena. En este sentido, fue el director del CEM, quien parado al lado del juez indígena, explicó al gobernador la forma en cómo opera el juzgado, todo esto captado y difundido por los medios de comunicación.

En esta dirección, Sharman y Gupta (2006), señalan que es precisamente en el ámbito de la representación que se produce el discurso del Estado. "Las representaciones culturales públicas y los performance sobre la condición del estado buscan dar forma a las percepciones sobre su naturaleza en las personas" (Ibídem, 2006:18) ${ }^{11}$. En este sentido, tenemos que dichas acciones no sólo permiten la continuidad de la institución, sino que reproducen su superioridad desde el mantenimiento de inequidades (Ibídem, 2006), como se desarrolla a continuación.

La instalación del juzgado indígena trajo consigo cambios en el campo jurídico regional, especialmente me interesa mencionar dos de ellos que han provocado gran confusión en la práctica local, el primero tiene que ver con las relaciones entre los jueces de paz -quienes se localizan en las comunidades y que tradicionalmente se han encargado de la resolución de conflictos-, y el juez indígena, ya que el TSJ no diferenció sus atribuciones. El segundo cambio, es el que tiene que ver con el estilo judicial en materia indígena que el TSJ busca imponer, el cual ha sido diseñado desde una lógica distinta a la práctica local, como mencioné anteriormente con los MARC, generando contradicciones entre distintas nociones de autoridad, justicia y derechos. 
Los jueces de paz son autoridades judiciales ubicadas en los pueblos de los distritos judiciales del Estado de Puebla. En regiones con población indígena, este cargo ha sido ocupado por dichos habitantes, lo que ha propiciado su inclusión dentro de prácticas culturales locales, como es el sistema de cargos, el cual conforma el sistema de organización comunitaria. La reforma judicial poblana al no haber tenido en consideración lo anterior, formuló disposiciones que no tomaron en cuenta la diferencia jurídica, y por tanto desconocieron su pluralidad, armonizando sin ningún contratiempo, a la justicia indígena con el proyecto de Estado. Esta situación es importante señalarla, ya que así como se crea una versión de justicia indígena en perfecta consonancia, se genera, por otra parte, una versión de justicia indígena ilegítima, para señalar a aquella que confronta al Estado por plantear otras nociones y valores normativos. Esta segunda versión es la que se ha vuelto susceptible de ser perseguida por la ley; Hale (2005) habla de la etnicidad disfuncional para referir a este tipo de representaciones creadas desde el poder.

En el vaivén entre estas representaciones de la justicia indígena oficializada y la justicia indígena disfuncional, nos encontramos con que diversas organizaciones del municipio de Cuetzalan, la Takachihualis, la Maseualsiuamej y el CADEM, iniciaron un proceso de apropiación del juzgado indígena, consiguiendo elaborar un proyecto de justicia propia, el cual resulta interesante, ya que dicho proyecto no confronta al Estado, pero tampoco lo rechaza o desconoce, sino que lo han elaborado dentro de los marcos del TSJ. Es así que a la par de la administración estatal y municipal del juzgado, miembros de estas organizaciones conformaron el consejo del juzgado indígena, desde donde iniciaron con diversas acciones para enfrentar la indefinición que la reforma judicial generó en el campo judicial en Cuetzalan.

Si bien se trata de un proceso en curso, con muchas limitaciones, ha sido fundamental para la formulación de un proyecto de justicia indígena propia. La conformación del consejo es un ejemplo que permite analizar los encuentros contradictorios de la modernidad con otros proyectos locales, asimismo, muestra el carácter dinámico de las sociedades indígenas y sus procesos de transformación cultural para responder a la política estatal. Entre las diversas acciones que han llevado a cabo los consejeros, me interesa mostrar 1) la manera en cómo han buscado posicionar la práctica de la justicia indígena, ante las disposiciones establecidas por el TSJ a partir de la instauración de los MARC, y 2) resolver la distribución jurisdiccional de la justicia indígena. Es decir, se trata de un proyecto que busca reelaborar las representaciones que median el proyecto estatal en materia indígena.

Un primer aspecto se relaciona con la instauración del juzgado indígena en la cabecera municipal de Cuetzalan, un espacio que se caracteriza por tener una mayoría de población mestiza, quienes son los que ocupan los puestos judiciales y político-administrativos, y los llevan a cabo bajo una lógica distinta a la de las comunidades. Dicha situación colocó al juzgado fuera del sistema de organización comunitaria, desprendiendo a esta nueva autoridad de las prácticas de prestigio locales, ante esta situación los consejeros han llevado a cabo ciertas acciones que podría decir, actualizan prácticas tradicionales. 
En las comunidades indígenas de Cuetzalan, los cargos de autoridad forman parte de un sistema de organización comunitaria, conocida como sistema de cargos de servicio, el cual funciona aproximadamente de la siguiente manera. Los distintos cargos están escalonados, el primero generalmente es el de topil, cuya función es prestar servicio de mensajero para los cargos de los siguientes escalafones. La persona que inicia en el sistema de cargos, de los cuales no se recibe ningún pago, va subiendo por cada uno de ellos; no existe un orden de sucesión estricto, pero a los cargos de mayor rango se accede cuando la persona tiene una trayectoria de ejercicio en cargos de servicio ${ }^{12}$.

La importancia de los cargos de servicio radica en que otorgan experiencia, respeto y legitimidad a las personas que han participado en ellos. Por ejemplo, la autoridad indígena encargada de impartir justicia, que es un cargo de mayor rango, va a ser reconocida por los habitantes de la comunidad por su trayectoria en los cargos. Por otra parte, la cercanía que se da entre los habitantes que viven en una comunidad, les permite saber si la participación de una autoridad fue buena, es decir, si cumplió con sus tareas, pero también, si no anduvo de borracho, si no estuvo involucrado en conflictos domésticos, etc. Es decir, existe "cierta vigilancia" del trabajo de las autoridades por la localidad

Bajo este contexto, los consejeros han visto la manera de vincular al juzgado indígena con prácticas tradicionales como las mencionadas, por ejemplo, el mismo consejo está integrado por alrededor de doce personas, hombres $y$ mujeres nahuas provenientes de varias comunidades, quienes además son reconocidos en sus localidades por haber ejercido cargos de servicio, de esta manera, han traído la representación de la población nahua al juzgado. En este sentido, en el consejo se asumieron dos responsabilidades centrales: elegir al juez indígena, tomando en cuenta su perfil de servicio comunitario, y "vigilar" su trabajo, como lo haría la colectividad en ambos casos. Las prácticas tradicionales vistas desde esta puesta en escena, representan una manera más dinámica, con agencia e historizada de entender la cultura, además de darle un sentido reivindicativo, ya que ha sido la forma en como las organizaciones locales confrontan y se ajustan a las nuevas condiciones de justicia que plantea el Poder Judicial.

Un segundo aspecto, tiene que ver con el impacto de la reforma judicial en la definición de la distribución jurisdiccional de la justicia indígena. Las acciones de los consejeros para hacer frente a lo anterior, en parte han sido posibles por la ilegibilidad que caracteriza el marco discursivo de la oficialización de la justicia indígena (Das, 2004). La ambigüedad discursiva entre la Ley Orgánica, el Código de Procedimientos Civiles, los funcionarios del TSJ y las autoridades municipales, ha permitido transformar su conceptualización. En esta dirección, el trabajo de los integrantes del consejo ha generado una práctica de justicia indígena distinta a la institucionalizada, lo cual muestra realidades y visiones distintas de justicia y derechos a las definidas por el TSJ. Si bien tampoco puede ser igual a la practicada al interior de las comunidades, definitivamente es más cercana a las necesidades de la población nahua.

Es así que el juez indígena, quien ha sido elegido por el consejo, continuó atendiendo 
como ha hecho en sus cargos pasados como juez de paz, asimismo, se ha buscado articular al juzgado con los jueces de paz, tratándose de implementar dos niveles de justicia conformado por un primero en la comunidad y un segundo en la cabecera municipal, lo cual podría constituir una jurisdicción indígena regional. A la par de esto, el juzgado cumple con el procedimiento que el Centro Estatal de Mediación les instruyó, el cual radica principalmente en el llenado de ciertos formatos. Esta combinación implica seguir en cierta medida el procedimiento de la mediación definido desde este centro, generando una compleja composición entre la práctica de la justicia indígena local y el proyecto de justicia del estado.

La instalación del juzgado indígena de Cuetzalan podríamos verlo como un avance para la justicia indígena, ya que se introdujo el uso de la lengua materna y prácticas de justica nahuas en la cabecera municipal de forma más evidente, sobrepasando los límites de la comunidad. Si bien no se ha logrado la articulación con los jueces de paz de manera continua, limitando la conformación de una jurisdicción indígena regional, es importante señalar que este proyecto estatal posicionó el tema de la justicia en las discusiones y demandas de las organizaciones y la población nahua del municipio, e incluso de municipios vecinos y otros grupos étnicos de Puebla.

\section{Apuntes de cierre}

La experiencia del juzgado indígena de Cuetzalan representa un estudio de caso, que proyectado de manera amplia, permite analizar cómo las políticas multiculturales del Estado continúan recreando y reforzando relaciones de exclusión y subordinación hacia los pueblos indígenas, cuya novedad, respecto de anteriores políticas indigenistas, es que institucionaliza estas relaciones de opresión en reformas constitucionales y legales bajo el discurso de la diversidad cultural. En contraste con lo anterior, esta experiencia muestra el carácter dinámico de las sociedades indígenas y sus procesos de transformación cultural para responder no sólo a políticas estatales abiertamente discriminatorias, sino también a políticas "sensibles" a la diferencia étnica

En este texto, buscamos acercarnos a este análisis desde las representaciones sociales presentes en la formulación e implementación de proyectos estatales en materia de justicia indígena, bajo este objetivo, evidenciamos cómo el discurso de reconocimiento de la diversidad cultural cobija la continuación de relaciones de discriminación y subordinación. Si bien esta observación contradice el espíritu multicultural, lo que hemos advertido en la experiencia del juzgado indígena, tanto en su conformación discursiva como en su implementación, es la continuidad de la lógica monocultural y la organización hegemónica del Estado, así como la reproducción de representaciones del indígena como inferior e incapaz de llevar a cabo sus propios asuntos. Es así que estas representaciones siguen vigentes para justificar el dominio discursivo del Estado, es decir, apoyan su legitimidad para definir a la cultura indígena.

Alcanzar estos resultados de estudio nos requirió incluir en el análisis la construcción discursiva del Estado y su relación con procesos nacionales y transnacionales; siguiendo 
la propuesta de Gupta (2006) observamos cómo el sistema interestatal es sujeto de transformaciones que provienen de acciones nacionales, pero también de presiones de otros Estados o de agencias internacionales que imponen cambios en el derecho local. La revisión de leyes, códigos y noticas publicadas por el TSJ, vistos como textos culturales, nos permitió ver la manera en cómo el Estado se representa y construye a sí mismo, pero también lo que excluye, omite y descalifica. En este sentido, advertimos cómo el Estado multicultural construye distintas versiones de justicia indígena, como aquellas en consonancia con el proyecto estatal y que alcanzan su institucionalización, y otras disfuncionales, que son ubicadas como una amenaza.

También nos concentramos en las prácticas de los burócratas, de esta forma contrastamos la relación entre una institución translocal como lo es el Poder Judicial, con sus oficinas y prácticas que lo instituyen localmente. Sharma y Gupta (2006) explican que el poder de las instituciones del Estado se constituye en la repetición de su representación en las prácticas diarias, sin embargo, los límites entre la potestad del Estado y el no-Estado, es una línea que en parte es dibujada por las representaciones y prácticas diarias de los burócratas en sus encuentros con los demás, así como desde las negociaciones que surgen entre estos. Es precisamente en este ámbito que nosotros ubicamos las posibilidades de llevar a cabo una práctica de justicia propia al interior del juzgado indígena.

A diferencia de otros movimientos o levantamientos armados indígenas en México, los cuales son más visibles en cuanto a sus expresiones críticas al Estado y sus demandas, en el caso del juzgado indígena de Cuetzalan, las organizaciones locales plantearon y pusieron en marcha acciones de reivindicación de la cultura nahua dentro de este espacio oficial. Lo que llama la atención en esta experiencia es la manera en cómo los integrantes del consejo y el juez han usado de manera estratégica su identidad a la hora de interactuar con los funcionarios del TSJ. Es así que en los eventos públicos los consejeros y el juez se presentan como el "indio permitido" (Hale, 2004), en consonancia con el proyecto estatal de justicia indígena, incluso llevan a cabo las disposiciones administrativas propias del CEM; lo anterior contrasta con las actividades cotidianas dentro del juzgado, donde la atención a los usuarios se realiza a partir de lógicas culturales nahuas, desde la concepción de los agravios hasta la dinámica de resolución de los problemas, ocultando su agenda por medio del uso de la lengua materna.

Si bien, actuar desde el interior de los marcos del Estado, podría verse como una opción limitada para la reproducción de la cultura indígena y sus derechos, en el caso del juzgado indígena nos encontramos con que algunas de las acciones impulsadas por las organizaciones involucradas rebasan las paredes del juzgado, como la búsqueda por implementar una jurisdicción indígena regional. Aún cuando esto último no se ha logrado, es cierto que los obstáculos que han enfrentado corresponden a situaciones que van mucho más allá de la reciente reforma en materia de justicia indígena y sus implicaciones en el campo jurídico regional, de aquí la necesidad de realizar estudios con perspectivas de larga data. 
Trabajar desde esta perspectiva, nos llevó a conocer la relación histórica entre el Estado y el pueblo nahua del municipio de Cuetzalan, misma que se ha caracterizado por la discriminación/ aceptación, lo cual ha permitido la continuidad de la cultura nahua sin fuertes acciones de prohibición por parte de las autoridades o de la población mestiza local (Terven, 2009). Visto así, el proceso de apropiación del juzgado, tiene la posibilidad de suceder dentro del mismo proyecto estatal, con concesiones realizadas por ambas partes. Revisar de manera crítica las políticas indigenistas y su relación con el actual contexto político multicultural permite advertir cambios, pero en nuestro caso nos interesó ver las continuidades, en particular las vinculadas con las representaciones discriminatorias hacia lo indígena, las cuales parecían ocultarse bajo un discurso de reconocimiento de la diversidad cultural.

Al abordar desde esta perspectiva el análisis de la relación entre el Estado y la población indígena -tratando de hacer una reflexión ampliapodemos decir que la política multicultural, al contrario de su promesa social, reinstituye de manera más radical las relaciones de subordinación y exclusión hacia los pueblos indígenas.

\section{Notas}

\footnotetext{
${ }^{1}$ A la fecha 26 de 31 Estados de la República Mexicana, han realizado reformas en materia indígena.

${ }^{2}$ Otras actividades agrícolas de productos comerciales son el café o la pimienta, y en menor escala cítricos y frutas, las familias también crían cerdos y guajolotes.

${ }^{3}$ Para conocer la revisión y análisis completo, ver: Maldonado \& Terven (2009).

${ }^{4}$ Proyección del Poder Judicial del Estado. Publicación del H. Tribunal Superior de Justicia del Estado de Puebla, Noviembre 2003, p. 12
}

\begin{abstract}
${ }^{5}$ Existen cinco juzgados indígenas en las cabeceras municipales de Quimixtlan y en Tlacotepec de Porfirio Díaz en el municipio de San Sebastián Tlacotepec, todos con población predominantemente nahua; en los municipios de Huehuetla con población totonaca y en Pahuatlan con población mayoritariamente otomí. ${ }^{6}$

${ }^{6}$ Acuerdo de Pleno, 14 de marzo de 2002.

${ }^{7}$ Acuerdo de Pleno, 14 de marzo de 2002.

${ }^{8}$ Acuerdo de Pleno, 14 de marzo de 2002.

${ }^{9}$ Capítulo Primero, artículo 832 del Nuevo Código de procedimientos Civiles del Estado de Puebla, p. 354.

${ }^{10}$ En un estudio realizado sobre la reforma en materia indígena en el estado de Querétaro por Terven, Vázquez \& Prieto (2013), igualmente señalan la manera en como la Ley de Derechos y Cultura de los Pueblos Indígenas del Estado, define una noción de justicia indígena que sólo se limita al ámbito comunitario, blindando así al aparato de justicia estatal para que prevalezca como la única lógica de derecho.

${ }^{11}$ Public cultural representations and performance of statehood crucially shape people's perceptions about the nature of the state (Sharman \&Gupta, 2006:18). Traducción propia.

${ }^{12}$ Existen casos en los que habitantes indígenas, por influencia de partidos políticos, por ejemplo, acceden a cargos, sin haber pasado por el sistema de cargos de servicio. En estos casos, puede suceder que los habitantes pidan su sustitución, como me ha tocado conocer, exigiendo se lleve a cabo el debido proceso que generalmente es la elección en asambleas comunitarias o por el cabildo. O simplemente no lo toman en cuenta y no acuden con él.
\end{abstract}

\section{Referencias bibliográficas}

Aguirre Beltrán, G. (1984) "Polémica Indigenista". América Indígena XLIV, 7-28.

Assies, W. (1999) "Pueblos indígenas y reformas del estado en América Latina". En Assies W. et. al. (eds.). El reto de la diversidad. Pueblos indígenas y reformas del Estado en América Latina, México: El Colegio de Michoacán.

Código de Procedimientos Civiles del estado de Puebla (2005) Colección de Leyes Mexicanas, serie: leyes del Estado de Puebla, México: Ed. Cajica.

Díaz-Polanco, H. (1996) Autonomía regional. La autodeterminación de los pueblos indios, México: Siglo XXI.

Díaz-Polanco, H. (2004) El canon Snorri. Diversidad cultural y tolerancia, México: UCM.

Das, V. (2004) "The signatura of the State: paradox of illegibility". En Das V. y Poole D. (editoras). Anthropology in the Margins of the State, Estados Unidos: School of American Research Press, James Currey Oxford.

Gupta, A. (2006) "Blurred boundaries: the discourse of corruption, the culture of politics, and the imagined State". En Sharma A. \& Gupta A. (eds). The anthropology of the State, Reino Unido: Blackwell Publishing. 
Hale, C. (2005) "Neoliberal Multiculturalism: the remaking of cultural rights and racial dominance in Central America", en POLAR: Political and legal Anthropology Review, vol. 28, No. 1, American Anthropological Association.

(2004) "Rethinking indigenous politics in the Era of the "Indio Permitido", reporte del Congreso de Norte América sobre América Latina NACLA, septiembre-octubre.

Hernández, A. Paz, S. Sierra, M. (Coords.) (2004) El Estado y los indígenas en tiempos del PAN: neoindigenismo, legalidad e identidad, México: Cámara de Diputados, Porrúa, CIESAS.

Ley Orgánica Del Poder Judicial. Edición oficial del 30 de enero de 2002, en Colección de Leyes Mexicanas, serie: leyes del Estado de Puebla, México: Ed. Cajica, 2005.

Maldonado, K. \& Terven, A. (2009) Los Juzgados Indígenas de Cuetzalan y Huehuetla. Vigencia y reproducción de los sistemas normativos de los pueblos de la Sierra Norte de Puebla, México: CDI.

Merry, S. (1988) "Legal pluralism", en Law and Society Review, 22 (5).

Proyección del Poder Judicial del Estado, Publicación del H. Tribunal Superior de Justicia del Estado de Puebla, Noviembre, 2003.

Oehmichen, C. (1999) Reforma del estado: política social e indigenismo en México, 1988-1996, México: UNAM, IIA.

Santos, B. (1999) La globalización del derecho. Los nuevos caminos de la regulación y la emancipación, Colombia: ILSA.

Sharma A. \& Gupta A. (eds). The anthropology of the State, Reino Unido: Blackwell Publishing.

Sieder, R. (editora) (2002) Multiculturalism in Latin America. Indigenous Rights, diversity and democracy. Reino Unido: Palgrave Macmillan.

Sierra, M. (2004) "Interlegalidad, justicia y derechos en la sierra norte de Puebla", en Sierra M. (coord.) Haciendo justicia. Interlegalidad, derecho y género en regiones indígenas, México: CIESAS-Porrúa.

Stavenhagen, R. (2002) "Indigenous people and the state in Latin America: an ongoing debate", en Sieder, R. (ed.) Multiculturalism in Latin America. Indigenous Rights, diversity and democracy, Reino Unido: Palgrave Macmillan.

Terven, A. (2009) Justicia indígena en tiempos multiculturales. Hacia la conformación de un proyecto colectivo propio: la experiencia organizativa de Cuetzalan, Tesis de doctorado en Antropología, México: Centro de Investigaciones y Estudios Superiores en Antropología Social (CIESAS).

Terven, A., Vázquez, A., \& Prieto, D. (2013) "La ciudad como espacio de multiculturalidad y ejercicio de derechos étnicos", en Vázquez, A. \& Prieto, D. (coords.) Indios en la ciudad. Identidad, vida cotidiana e inclusión de la población indígena en la metrópoli queretana, México: INAH, UAQ, CONACyT.

Vázquez, A. (2011) Intención y sospecha. Acciones, discursos y políticas dirigidas hacia los pueblos indígenas de México, Tesis de doctorado, España: Universidad Pablo de Olavide.

Villoro, L. (1996) Los grandes momentos del indigenismo en México, México: El Colegio de México. 